\title{
Generation and Propagation of Normal and Abnormal Pacemaker Activity in Network Models of Cardiac Sinus Node and Atrium
}

\author{
RAIMOND L. WINSLOW, ${ }^{*}$ DONGMING CAI, $\uparrow$ ANTHONY VARGHESE $\ddagger$ and YING-CHENG LAI*
}

*Department of Biomedical Engineering, The Johns Hopkins University School of Medicine, Baltimore, MD 21205, USA †Biomedical Engineering Program, The University of Minnesota, Minneapolis, MN 55455, USA and $\ddagger$ Army High Performance Computing Research Center, The University of Minnesota, Minneapolis, MN 55455, USA

\begin{abstract}
Effects of cell-to-cell coupling conductance on dynamics of sinus node cells are examined. Cell models are biophysically detailed, and are based on the kinetic equations developed by Noble et al. [Neuronal and Cellular Oscillators, edited by J. W. Jacklet. Marcel Deckker, New York (1989).] Resistively coupled cell pairs show five regimes of behavior as a function of coupling conductance: (1) independent oscillation for $G_{\mathfrak{c}}<1 \mathrm{pS}$; (2) primarily quasiperiodic oscillation for $1 \leqslant G_{c}<116 \mu \mathrm{S}$; (3) windows of periodic behavior which undergo period doubling bifurcation to chaos for $116 \leqslant G_{c}<212 \mathrm{pS}$; (4) frequency entrainment for $G_{\mathrm{c}} \geqslant 212 \mathrm{pS}$; (5) waveform entrainment for $G_{\mathrm{c}} \geqslant 50 \mathrm{nS}$. Thus, only $4-5$ gap junction channels are required for frequency entrainment. This is shown to also be the case for large networks of sinus cells modeled on the Connection Machine CM-5. A biophysically detailed two-dimensional network model of the cardiac atrium has also been implemented on the CM-5 supercomputer. The model is used to study effects of spatially localized inhibition of the $\mathrm{Na}-\mathrm{K}$ pump. Na overloading produced by pump inhibition can induce spontaneous, propagating ectopic beats within the network. At a cell-to-cell coupling value yielding a realistic plane wave conduction velocity of $60 \mathrm{cms}^{-1}$ pump inhibition in small regions of the network containing as few as 1000 cells can induce propagating ectopic beats.
\end{abstract}

\section{INTRODUCTION}

The pacemaker of the normal heart is the sinoatrial (SA) node, a thin sheet of tissue located in the right atrium near the base of the superior vena cava. Each cell within the node is an electrochemical oscillator, exhibiting a distinct oscillation frequency and waveform $[1,2]$. Oscillation properties vary as a function of cell location within the node, with peripheral cells exhibiting higher oscillation frequencies and larger overshoot potentials, maximum diastolic potentials, and upstroke velocities than central cells [1]. It is likely that these variations result from systematic differences in the relative density of ion channels in cell membranes as a function of location within the node. Random differences in the number of cell-membrane ion channels at any particular location within the node also contribute to variability of oscillation frequency and waveform.

Despite these differences in oscillation properties, the SA node generates a periodic electrical wave of depolarization which spreads throughout the heart, determining its rate of contraction. The process by which cells within the SA node lock on to a common uscillation frequency is known as frequency entrainment. Early morphological studies demonstrated the presence of gap junctions between neighboring SA node cells, suggesting the hypothesis that frequency entrainment occurs as a result of ionic current flow through these junctions. Electrophysiological studies of frequency entrainment using embryonic heart-cell aggregates demonstrated that, when aggregates beating at their own intrinsic 
frequency were brought into physical contact, synchronization to a common frequency followed within minutes [3-5]. The transition to electrical synchronization was accompanied by a decrease in resistance of the junctional area between the aggregates. Mechanism of synchronization between SA node cells were studied further using the sucrose gap technique $[6,7]$. These experiments demonstrated that electrical coupling results in mutual entrainment with both pacemakers beating at simple harmonic or more complex ratios depending on the extent of coupling and intrinsic frequency of the two pacemaker regions.

Frequency entrainment has also been investigated through the use of models. Torre [8] studied properties of resistively coupled cell networks in which individual cells were modeled using the Bonhoeff-Van der Pol (BVP) equations. The model was used to prove the existence of a structurally stable periodic solution of the coupled systems at low values of coupling resistance, demonstrating that electrical coupling alone could account for frequency entrainment. In 1980, Yanagihara and his colleagues developed the first SA node-cell model based on voltage-clamp data obtained from multicellular preparations [9]. Michaels et al. [10] used these models in studies of frequency entrainment of resistively coupled cells pairs to demonstrate that phase-dependent interactions underlie synchronization of oscillation frequency. More recently, Winslow et al. [11, 12] and Cai et al. [13] have used the Noble single sinus cell model [14-16] to demonstrate that only a few gap-junction channels between cells are sufficient to account for frequency entrainment in both cell pairs and large networks.

While there is substantial morphological, electrophysiological, and modeling evidence demonstrating that electrical coupling of SA node cells by gap junctions underlies frequency entrainment within the SA node, there is as yet no clear understanding of how the magnitude of coupling affects cell dynamics. Dependence of cell dynamics on coupling strength has been studied using highly simplified pulse-coupled oscillator models [17-19]. This dependence has not been explored using more biologically detailed oscillator models. Differences in the dynamics of such models are expected; Brailove has reported that non-periodic motion does not exist for pulse-coupled oscillators [19], whereas quasiperiodic motion has been reported in resistively coupled, biologically realistic models of SA node cells [13]. We will present a detailed bifurcation analysis of the dependence of SA node-cell dynamics on the magnitude of gap-junction coupling conductance.

Pacemaker sites may also develop outside the SA node. Such sites are known as ectopic foci. One way in which ectopic foci may occur is through elevation of intracellular sodium (Na) subsequent to $\mathrm{Na}-\mathrm{K}$ pump inhibition. Consequences of $\mathrm{Na}-\mathrm{K}$ pump inhibition were first studied by Ferrier and Moe [20] and Lederer and Tsien [21], who inhibited pump activity in Purkinje cells using strophanthidin. A damped oscillatory inward current (the transient inward current, or TIC) was observed in voltage-clamped cells during repolarizing steps applied following depolarizing clamps [21]. In some cases, the TIC was sufficiently large to produce afterpotentials or triggered activity. Kass et al. [22] proposed the idea that the TIC was generated by calcium ( $\mathrm{Ca}$ ) efflux through the $\mathrm{Na}-\mathrm{Ca}$ exchanger in response to oscillatory release of $\mathrm{Ca}$ ions from intracellular stores. This hypothesis was strengthened following direct measurement of oscillation of intracellular $\mathrm{Ca}$ concentration $[\mathrm{Ca}]_{\mathrm{i}}$ in voltage-clamped cardiac cells following $\mathrm{Na}-\mathrm{K}$ pump inhibition [23].

Development of Purkinje [24], sinus node [15], atrial [25, 26], and ventricular cell models [27-29], which include voltage-dependent ionic currents, $\mathrm{Na}-\mathrm{K}$ and $\mathrm{Na}-\mathrm{Ca}$ pump/ exchanger currents, time-dependent changes in internal ion concentration, Ca-buffering, and sequestration-release of $\mathrm{Ca}$ from sarcoplasmic reticulum, has made possible the numerical simulation of the short and long term effects of the $\mathrm{Na}-\mathrm{K}$ pump blockade. The models have been used to reproduce a range of experimental observations on effects of 
$\mathrm{Na}-\mathrm{K}$ pump inhibition including afterdepolarizations and oscillation of $[\mathrm{Ca}]_{\mathrm{i}}$ during $\mathrm{Na}$ overload [27,28]. Varghese and Winslow [30] used bifurcation analyses to investigate stability properties of the system of differential equations describing the Ca subsystem in the DiFrancesco-Noble Purkinje fibre model under voltage-clamp conditions. In these analyses, internal sodium concentration $[\mathrm{Na}]_{i}$ was treated as a variable control parameter. Stability properties of asymptotic solutions were studied as a function of $[\mathrm{Na}]_{\mathrm{i}}$. A single stable fixed point corresponding to a low internal $\mathrm{Ca}$ concentration was demonstrated at low $[\mathrm{Na}]_{\mathrm{i}}$ values. As $[\mathrm{Na}]_{i}$ was increased, a critical point was reached at which the fixed point became unstable, giving rise to a stable periodic oscillation of $[\mathrm{Ca}]_{i}$ through a supercritical Hopf bifurcation. Stable periodic orbits existed for $[\mathrm{Na}]_{\mathrm{i}}$ levels in the range of $14-22 \mathrm{mM}$.

These results have demonstrated that conditions leading to $\mathrm{Na}$ overload in single cardiac cells have the potential to induce abnormal automaticity. We will extend these analyses to networks of atrial cells. We will demonstrate that $\mathrm{Na}$ overloading in small localized regions of atrial cells can lead to the development of an ectopic focus. We explore the relationship between the magnitude of cell-to-cell coupling and the number of $\mathrm{Na}$-overloaded cells required to generate propagating ectopic beats in both two- and three-dimensional atrial network models.

\section{METHODS}

\subsection{Single-cell models}

The single SA node-cell model used in these studies was developed by Noble and his colleagues, and is based directly on voltage-clamp data obtained from isolated cells [14-16]. The single atrial cell model was developed by Earm and Noble [26], as based on previous work by Hilgemann and Noble [25], and is included in the OXSOFT Heart Version 3.8 software distribution [16].

The general form of the system of differential-algebraic equations defining both models is:

$$
\begin{gathered}
\dot{V}_{\mathrm{t}}=-\frac{1}{C_{\mathrm{m}}}\left[\sum_{k} I_{k}\left(V_{\mathrm{t}}\right)+\sum_{k} P_{k}\left(C_{\mathrm{i}}^{m}, C_{\mathrm{i}}^{n}, C_{\mathrm{o}}^{m}, C_{\mathrm{o}}^{n}, V_{\mathrm{t}}\right)\right] \\
I_{k}\left(V_{\mathrm{t}}\right)=\left(V_{\mathrm{t}}-E_{k}\right) G_{k}^{\max } m_{k}\left(V_{\mathrm{t}}\right)^{p_{k}} h_{k}\left(V_{\mathrm{t}}\right)^{q_{k}} \\
\dot{m}_{k}\left(V_{\mathrm{t}}\right)=\alpha_{k}^{m}\left(V_{\mathrm{t}}\right)\left[1-m_{k}\left(V_{\mathrm{t}}\right)\right]-\beta_{k}^{m}\left(V_{\mathrm{t}}\right) m_{k}\left(V_{\mathrm{t}}\right) \\
\dot{h}_{k}\left(V_{\mathrm{t}}\right)=\alpha_{k}^{h}\left(V_{\mathrm{t}}\right)\left[1-h_{k}\left(V_{\mathrm{t}}\right)\right]-\beta_{k}^{h}\left(V_{\mathrm{t}}\right) h_{k}\left(V_{\mathrm{t}}\right) \\
\dot{C}_{\mathrm{i}}^{k}=-\sum_{k} I_{k}^{\text {total }} / z_{k} F V_{\mathrm{i}} .
\end{gathered}
$$

Equation (1) gives the time rate of change of membrane potential $V_{\mathrm{t}} . C_{\mathrm{m}}$ is total cell membrane capacitance, $I_{k}\left(V_{\mathrm{t}}\right)$ is the $k$ th outward membrane current, $P_{k}\left(C_{\mathrm{i}}^{m}, C_{\mathrm{i}}^{n}, C_{\mathrm{o}}^{m}, C_{\mathrm{o}}^{n}, V_{\mathrm{t}}\right)$ is an algebraic function specifying the $k$ th outward pump/ exchanger current, and $C_{\mathrm{i}}^{m}$ and $C_{\mathrm{o}}^{m}$ are internal and external concentrations of the $m$ th ionic species. Equations (2) -(4) specify the general form of voltage-gated membrane currents. $E_{k}$ is the reversal potential of the ion(s) to which the $k$ th membrane conductance is permeable, $G_{k}^{\max }$ is the peak conductance, and $m_{k}\left(V_{t}\right)$ and $h_{k}\left(V_{\mathrm{t}}\right)$ are activation and inactivation factors. Variables $m_{k}$ and $h_{k}$ are solutions of nonlinear ordinary differential equations (3)-(4). The functions $\alpha_{k}^{m, h}\left(V_{\mathrm{t}}\right)$ and $\beta_{k}^{m, h}\left(V_{\mathrm{t}}\right)$ are forward and backward voltage-dependent rate constants for the activation and inactivation variables $m_{k}$ and $h_{k}$. 
Equation (5) specifies the time rate of change of the intracellular concentration of the $k$ th ionic species (either $\mathrm{Na}, \mathrm{Ca}$, or $\mathrm{K}$ ). Extracellular concentrations are assumed to remain constant. In equation (5), $I_{k}^{\text {total }}$ is the total outward current, $V_{\mathrm{i}}$ the intracellular volume, $F$ Faraday's constant, and $z_{k}$ the valence of the $k$ th ionic species. In the case of calcium, $I_{\mathrm{Ca}}^{\text {total }}$ includes the sarcoplasmic reticulum uptake and release currents. Effects of Ca-buffering by troponin and calmodulin are also included in the atrial cell model.

\subsection{Coupled SA node cell pairs}

In these studies, two model SA node cells were coupled by a linear conductor $G_{\mathrm{c}}$. Oscillation frequencies and waveforms of the two cells were adjusted by identifying a minimum set of membrane current parameters, which when varied, could account for differences in cell oscillation properties observed within the SA node by Kodama and Boyett $[1,13]$. Maximum diastolic potential (MDP), overshoot potential (OP), oscillation period (P), and upstroke velocity (UV) for one cell were set to $-78 \mathrm{mV}, 28 \mathrm{mV}, 276 \mathrm{~ms}$, and $25 \mathrm{~V} \mathrm{~s}^{-1}$, respectively. This cell therefore modeled rapidly oscillating cells found within the SA node [1]. MDP, OP, P, and UV values in the remaining cell were set to $-58 \mathrm{mV}$, $4 \mathrm{mV}, 347 \mathrm{~ms}$, and $1.2 \mathrm{~V} \mathrm{~s}^{-1}$, respectively. This cell modeled the slowest-beating SA node cells. The coupled system of differential equations defining the two-cell model were integrated using a fourth-order adaptive step Runge-Kutta algorithm.

Asymptotic dynamics of cell pairs were studied as a function of coupling conductance using: (a) phase portrait; (b) power spectrum; (c) delayed coordinate embedding [31-35]; and (d) Poincaré surface of section analyses [34-36]. Analyses were conducted over the range of coupling values from 1 to $240 \mathrm{pS}$, typically in steps of $2 \mathrm{pS}$ (except near bifurcation points). Data for these analyses were generated by imposing a set of initial conditions, then integrating the state equations until a steady-state was achieved (typically $100 \mathrm{~s}$ ). State variables were then computed over an additional 100 -s window at a sampling interval of $50 \mu \mathrm{s}$. The resulting time series were used to reconstruct attractors within a $k$-dimensional embedding space. The necessary dimension of the embedding space was determined by incrementing $k$ until no new structures were evident in the attractor. For the parameter regime studied, $k=3$ appears sufficient to describe the dynamics.

\subsection{Network analyses}

Large-scale SA node and atrial networks were modeled as either two- or threedimensional lattices of resistively coupled cells. Coupling conductance $G_{\mathrm{c}}$ was set to a uniform value throughout the mesh. Network models were implemented on the Thinking Machines massively parallel CM-5 supercomputer in CMFORTRAN (Version 2.1 Beta 0.1 ). Details of implementation have been described previously [12]. Briefly, sub-grids of cells were mapped onto separate vector-processing units of the CM-5 and integrated using an adaptive step Merson-modified fourth-order Runge-Kutta algorithm [37]. Absolute error tolerance was set to $10^{-6}$.

\section{RESULTS}

\subsection{Normal pacemaking}

3.1.1. Dynamics of cell pairs. Figure 1(A)-(D) shows representative oscillation patterns of two SA node cells at coupling conductances of $0.1,70,1000$, and 50,000 pS. In Fig. 1(A) ( $0.1 \mathrm{pS}$ coupling), the two cells oscillate with the same intrinsic period and waveform as 
A. $\mathrm{Gc}=0.1 \mathrm{pS}$

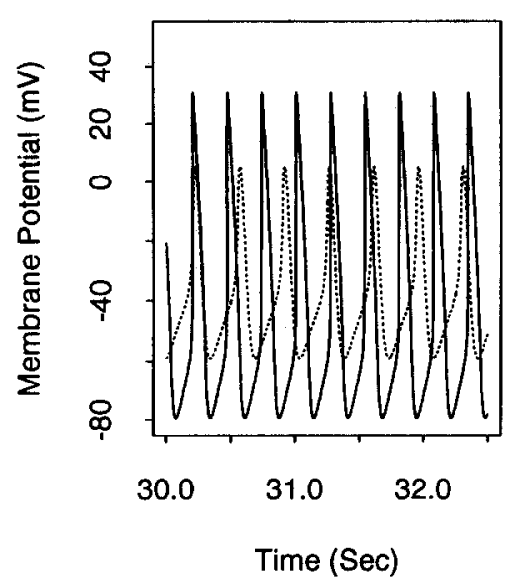

C. $\mathrm{Gc}=1 \mathrm{nS}$

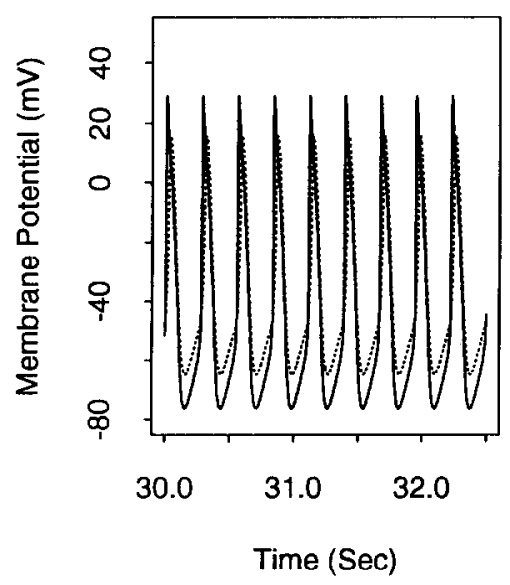

B. $\mathrm{Gc}=70 \mathrm{pS}$

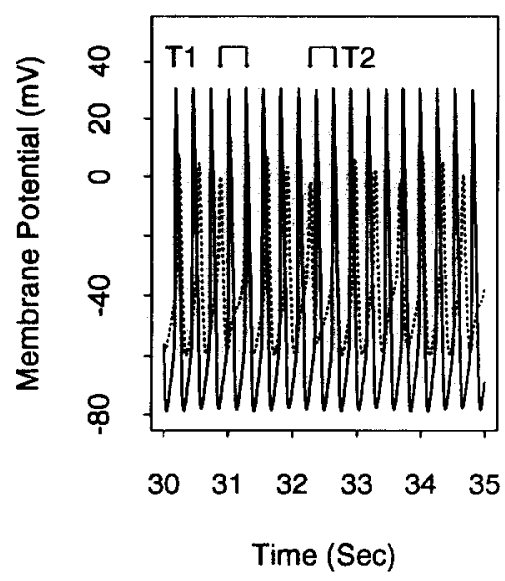

D. $\mathrm{Gc}=50 \mathrm{nS}$

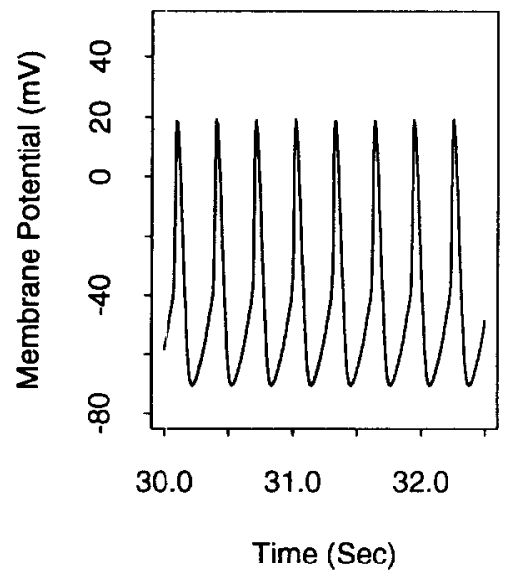

Fig. 1. Oscillation waveforms of the rapidly (solid line) and slowly (dotted line) oscillating SA node cell as a function of coupling conductance. Coupling conductance values are: (A) $0.1 \mathrm{pS}$; (B) $70 \mathrm{pS}$; (C) $1 \mathrm{nS}$ and (D) $50 \mathrm{nS}$.

they do in isolation. In Fig. 1(B) (70 pS coupling), oscillation waveforms are more complex. These effects are present in the responses of both cells, but are seen most clearly in those of the slower beating cell (dashed line). Oscillation of this cell appears to be modulated by a slowly varying envelope, reflected in the slow, repetitive variation of the MDP and UV amplitude. Oscillation period also varies in time, from a maximum of $399 \mathrm{~ms}$ during interval $\mathrm{T} 1$ to $387 \mathrm{~ms}$ interval T2. In Fig. 1(C), coupling conductance is increased to $1 \mathrm{nS}$. In this case, the oscillation period of both cells is about $290 \mathrm{~ms}$ the cells are frequency entrained. However, cell waveforms are clearly different, being phase-shifted relative to one another. This phase shift results from the fact that both the inward Ca- and Na-current magnitudes are smaller in the more slowly beating cell, resulting in a slower upstroke velocity. Figure 1(D) shows oscillation waveforms when coupling is increased to $50 \mathrm{nS}$. In this case, both oscillation frequency and waveform are identical in the two cells. 
The data of Fig. 1 suggest that there are at least four dynamic regimes as a function of coupling conductance: (a) a regime of independent oscillation at low coupling values; (b) a regime of complex dynamics at slightly higher coupling; (c) a regime of frequency, but not waveform entrainment; and (d) a regime of both frequency and waveform entrainment at high coupling values. This possibility is explored further in Fig. 2, which shows values of the oscillation period (Fig. 2(A)), overshoot potential (Fig. 2(B)), and MDP (Fig. 2(C)) as a function of coupling conductance (abscissa; in units of the base 10 logarithm of

A

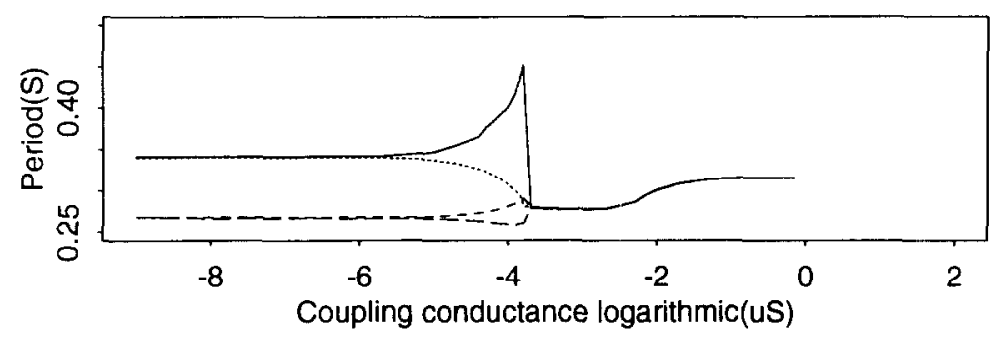

B

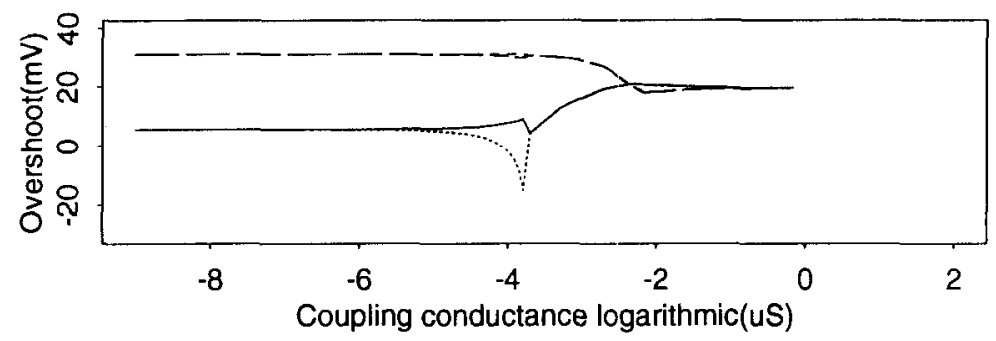

C

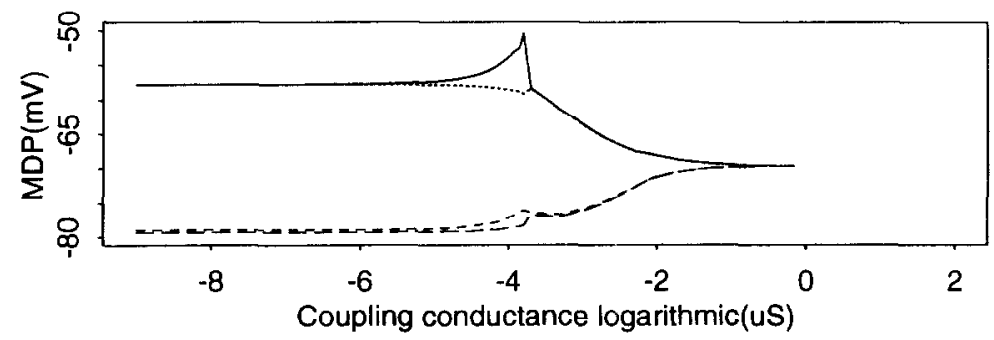

Fig. 2. (A) Oscillation period (ns), (B) overshoot potential (mV), and (C) maximum diastolic potential (mV) as a function of coupling conductance. In each panel, solid and dotted lines show the maximum and minimum values (respectively) of the period, UV, and MDP for the rapidly oscillating cell. The dashed and long lines show corresponding values for the slower oscillator. The abscissa is the base 10 logarithm of the coupling conductance in $\mu \mathrm{S}$. 
conductance in $\mu \mathrm{S}$ ). Four curves are shown in each plot. Solid and dotted curves are derived from responses of the more slowly oscillating cell, and show maximum (solid line) and minimum (dotted line) values of the quantity displayed on the ordinate during the time course of the oscillation. Short and long dashed lines plot maximum and minimum values of the same response measure for the more rapidly oscillating cell.

Inspection of these data shows that for coupling values less than approximately $1 \mathrm{pS}$, changes in coupling conductance have little effect on the oscillation period of either cell. Both beat at their intrinsic periods of approximately 347 and $276 \mathrm{~ms}$. Within this range, variation of coupling also has little effect on either the overshoot (Fig. 1(B)) or maximum diastolic potentials (Fig. 1(C)). This indicates that independent oscillation of coupled cells occurs for coupling values less than approximately $1 \mathrm{pS}$.

Divergence of the solid and dotted lines at coupling values greater than $1 \mathrm{pS}$ indicates the onset of complex oscillation of the form shown in Fig. 1(B). Both the oscillation period (Fig. 2(A)), overshoot potential (Fig. 2(B)), and maximum diastolic potential (Fig. 2(C)) vary during oscillation. This variation is largest at a coupling value slightly greater than $100 \mathrm{pS}$, and ceases to exist as coupling conductance approaches a value slightly larger than $200 \mathrm{pS}$. At this value of coupling, the oscillation period of the two cells takes on a common value of $290 \mathrm{~ms}$ (Fig. 2(A)). The overshoot and maximum diastolic potentials remain different despite the fact that the two cells are frequency entrained. As coupling conductance is increased, the overshoot and maximum diastolic potential of the two cells gradually become closer, and are in approximate agreement for coupling values greater than about $50 \mathrm{nS}$. These data suggest a more precise partitioning of system dynamics as a function of coupling conductance $G_{\mathrm{c}}$ : (a) independent oscillation for $G_{\mathrm{c}}<1 \mathrm{pS}$; (b) complex oscillation for $1 \leqslant G_{c}<200 \mathrm{pS}$; (c) frequency, but not waveform entrainment for $G_{\mathrm{c}} \geqslant 200 \mathrm{pS}$; and (d) both frequency and waveform entrainment for $G_{\mathrm{c}} \geqslant 50 \mathrm{nS}$.

Figure 3 shows the results of analyses of the dynamics within the complex regime. In this case, the coupling conductance was set to $70 \mathrm{pS}$. Figure 3(A) shows a phase plane plot of internal calcium concentration $[\mathrm{Ca}]_{i}$ (abscissa) and membrane potential (ordinate) for the slower oscillator. Inspection of the plot shows that no particular trajectory is repeated during successive action potentials, or if they are repeated, the period is so long that it cannot be resolved. Note that the magnitude of $[\mathrm{Ca}]_{i}$ has an overall fluctuation of about a factor of two. Figure 3(B) shows the power spectrum of the membrane potential of this same cell, computed with a spectral resolution of $0.05 \mathrm{~Hz}$. The arrows point to frequency components that can be accounted for as integer combinations of the intrinsic oscillation frequencies of the cells, the defining property of a quasiperiodic signal. These components account for a substantial portion of the energy in the voltage waveform. Figure 3(C) shows an attractor reconstruction using the method of Packard et al. [31] and Takens [32]. The plot was generated from the set of state variables $V\left(t_{k}\right), V\left(t_{k}-\tau\right), V\left(t_{k}-2 \tau\right)$, where $\tau$ is a time-lag ( $\tau=25 \mathrm{~ms}$ in our studies). The attractor is clearly toroidal in structure. Figure $3(\mathrm{D})$ is a two-sided Poincaré section generated by cutting the flow in Fig. 3(C) with a hyperplane positioned at $V\left(t_{k}\right)=-10 \mathrm{mV}$. Points of intersection of the flow with the plane generate two closed curves, demonstrating that dynamics of the coupled cell pair at this level of coupling evolve on the surface of a torus, and are therefore quasiperiodic.

In order to gain a more complete understanding of the dynamics of the coupled cells as a function of coupling conductance, we have reconstructed attractors for coupling values in the range of 1 to $240 \mathrm{pS}$. Figure 4 shows a bifurcation diagram that summarizes these results. The bifurcation diagram was computed by projecting one-sided Poincaré sections similar to those of Fig. 3(D) onto the $V(t-\tau)$ axis for each value of $G_{\mathrm{c}}$. With this approach, a period- $k$ orbit projects to $k$ discrete points. For quasiperiodic motions, projections will form a one-line segment (if the sampling of points on the surface of the 
A

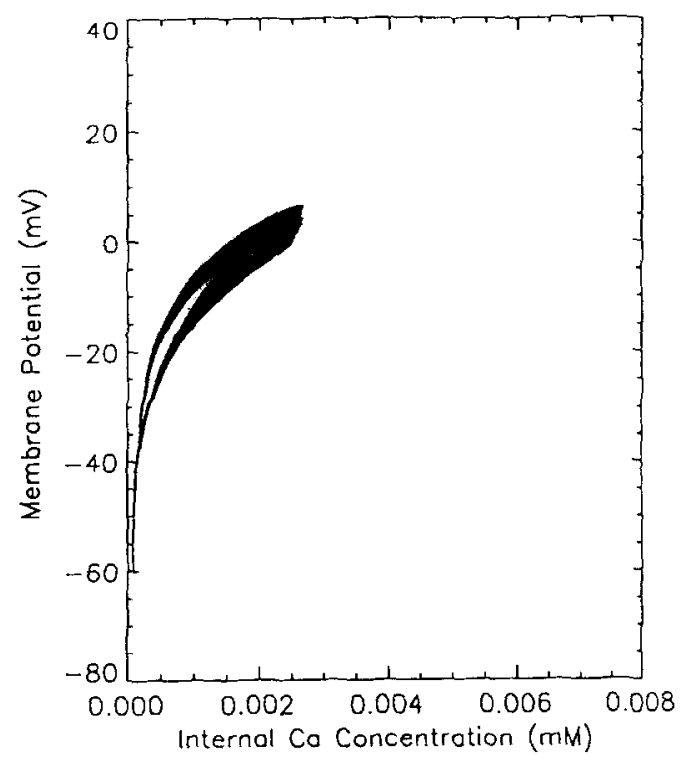

C

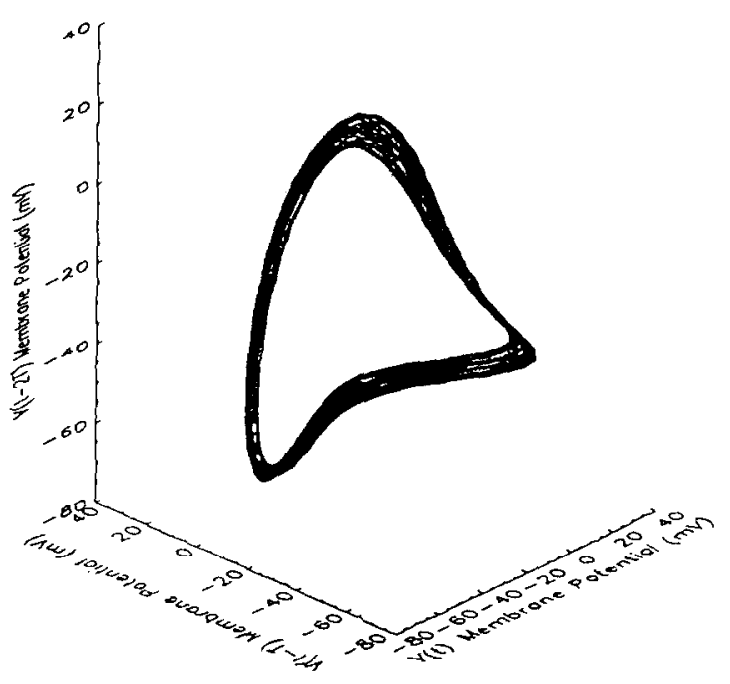

B

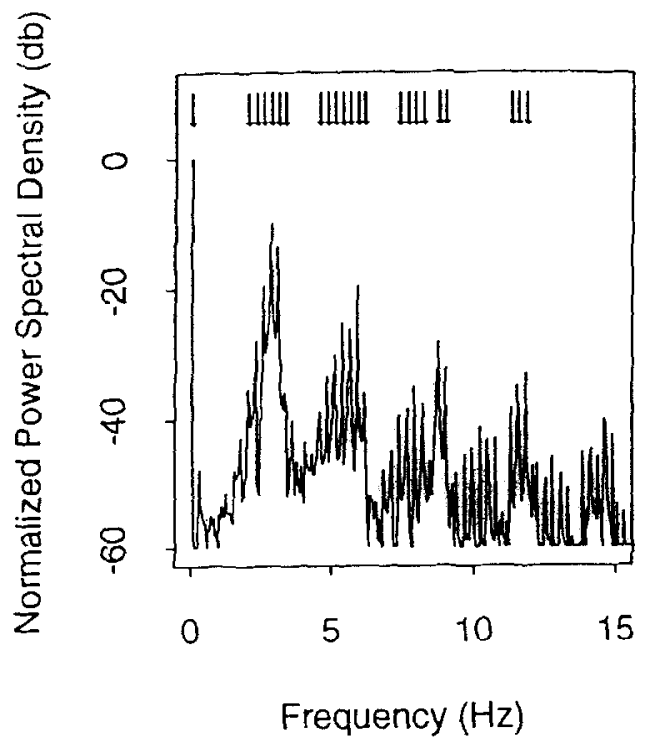

D

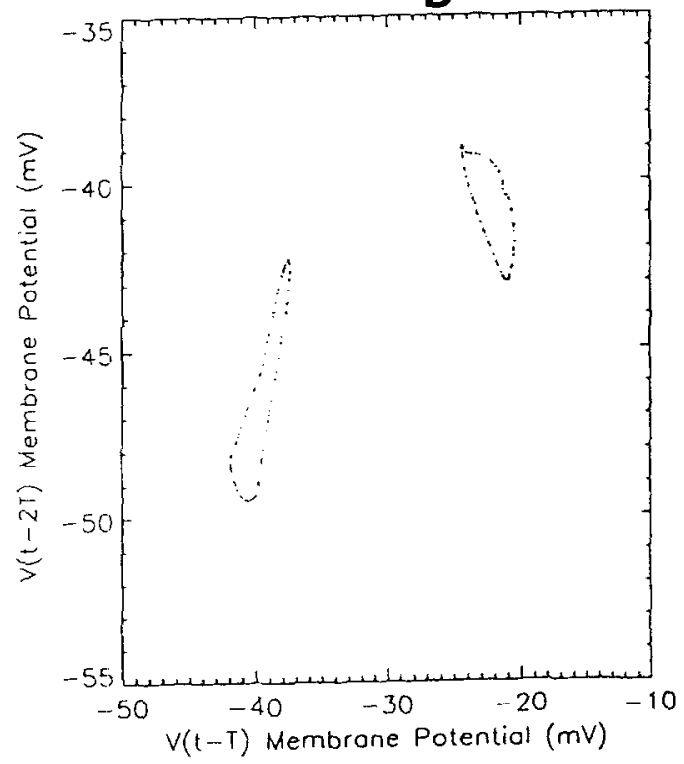

Fig. 3. Analyses of the oscillation properties of the more slowly oscillating cell at a coupling conductance of $70 \mathrm{pS}$. (A) V-Ca phase plane; (B) normalized power spectrum; (C) attractor reconstruction using three delayed coordinates with $\tau=25 \mathrm{~ms}$; (D) Poincaré section computed from (C) by intersecting the flow with a plane located at $V(t)=-10 \mathrm{mV}$.

torus is sufficiently dense). As will be seen, projections for chaotic motions will typically form a number of short line segments.

Figure 4 demonstrates three major dynamic regimes corresponding to $1 \leqslant G_{\mathrm{c}}<116 \mathrm{pS}$ (regime 1), $116 \leqslant G_{c}<212 \mathrm{pS}$ (regime 2), and $G_{\mathrm{c}} \geqslant 212 \mathrm{pS}$ (regime 3). For most $G_{\mathrm{c}}$ values in regime 1 , motion is quasiperiodic. However, there also exist conductance values for 


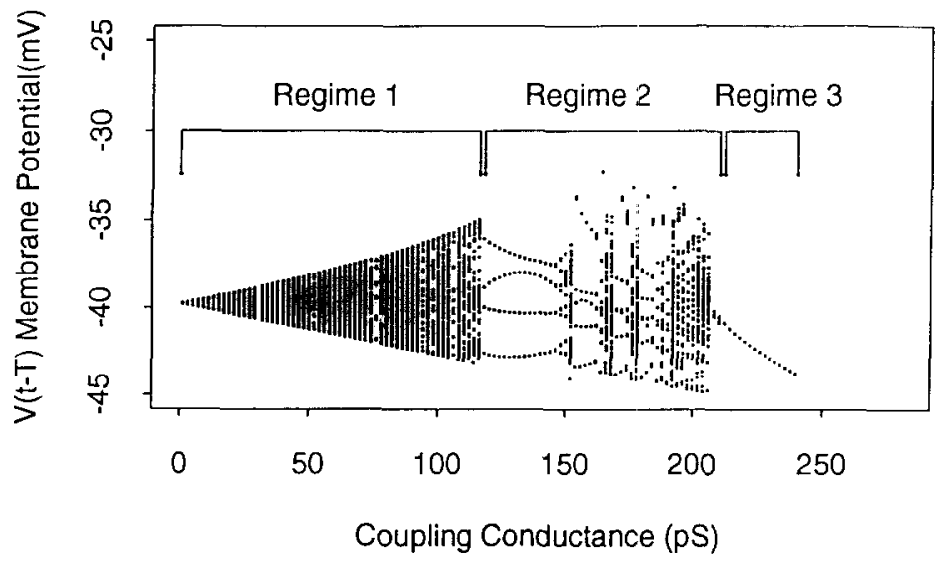

Fig. 4. Bifurcation diagram computed by projecting one-sided Poincaré sections onto the $V(t-\tau)$ axis (ordinate) at different coupling conductance values $G_{\mathfrak{c}}$ (abscissa).

which the attractor is periodic. For instance, a period- 6 attractor occurs at $G_{\mathrm{c}}=76 \mathrm{pS}$. The occasional coupling values giving rise to periodic attractors are interspersed between those producing quasiperiodic attractors. This is known as the Arnold's tongue phenomenon, and has been studied extensively for the family of circle maps [38].

At $G_{\mathrm{c}} \approx 116 \mathrm{pS}$, the quasiperiodic attractor bifurcates to a period-4 attractor. As $G_{\mathrm{c}}$ is increased, the system undergoes a period-doubling cascade. This is illustrated in Fig. 5 which shows Poincaré sections at different conductance valucs to identify period-4 (Fig. 5(A)), period-8 (Fig. 5(B)), and period-16 (Fig. 5(C)) attractors. The surface of section computed from the attractor at $G_{\mathrm{c}}=152 \mathrm{pS}$ (Fig. 5(D)) has a complex shape. Computation of the largest Lyapunov exponent yields a value of 0.2 . It therefore appears that this sequence of bifurcations is a period-doubling route to chaos.

As $G_{\mathrm{c}}$ is increased beyond $152 \mathrm{pS}$, period-5, 6, and 7 windows giving rise to additional period-doubling cascades are observed. Such windows have been observed in other chaotic dynamical systems such as the logistic and Henon maps [39]. Finally, for $G_{\mathrm{r}} \geqslant 212 \mathrm{pS}$ (regime 3), the attractor becomes a period-1, signifying frequency entrainment of the two cells.

3.1.2. Dynamics of large SA node network models. Results of the cell-pair studies indicate that low values of coupling (as few as 4-5 gap junction channels, assuming a conductance of $50 \mathrm{pS}$ per channel [40]) are sufficient to account for frequency entrainment of cell pairs. In order to investigate properties of frequency entrainment in larger $N \times N$ networks of resistively coupled cells, parameters of node cells within the mesh were varied randomly to produce two-dimensional network models in which each cell has a different oscillation frequency in the range of 276 to $347 \mathrm{~ms}$. Uniform coupling and initial conditions were imposed on the network, and the state equations were integrated in parallel on the CM-5.

Figure 6 shows plots of instantaneous period at $t=2.515 \mathrm{~s}$ after onset of the simulation. Instantaneous period at time $t$ is computed for each cell in the mesh by setting a threshold voltage equal to the membrane potential $V(t)$, computing the time at which the last threshold crossing of the same sign occurred. Four plots are shown, each displaying instantaneous period at the same time as in Fig. 6(A), but with different uniform coupling values. With no coupling between cells (Fig. 6(A)), there is a wide dispersion of oscillation periods throughout the mesh. The mean and standard deviation of the oscillation period is 
A. 146 ps

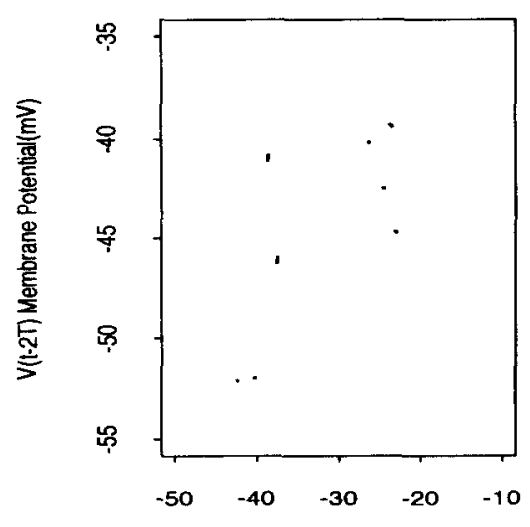

$V(t-T)$ Membrane Potential(mV)

C. $148.3 \mathrm{ps}$

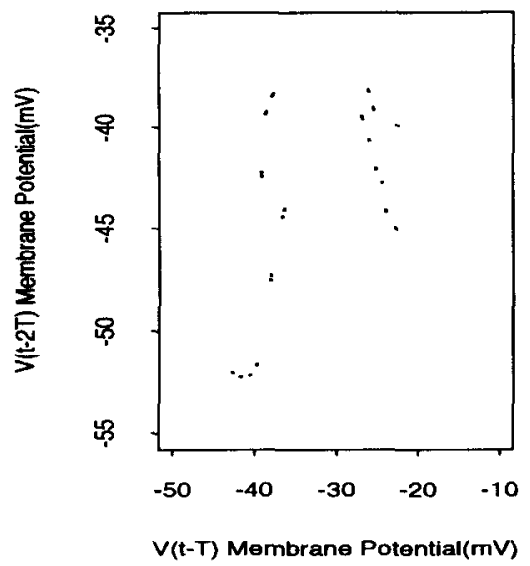

B. $148 \mathrm{ps}$

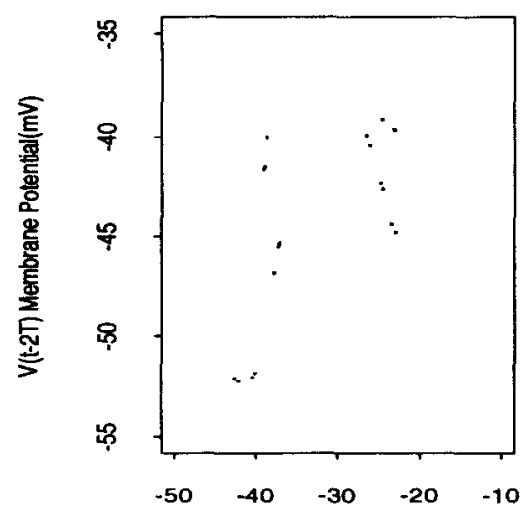

$\mathrm{V}(\mathrm{t}-\mathrm{T})$ Membrane Potential(mV)

D. $152 \mathrm{ps}$

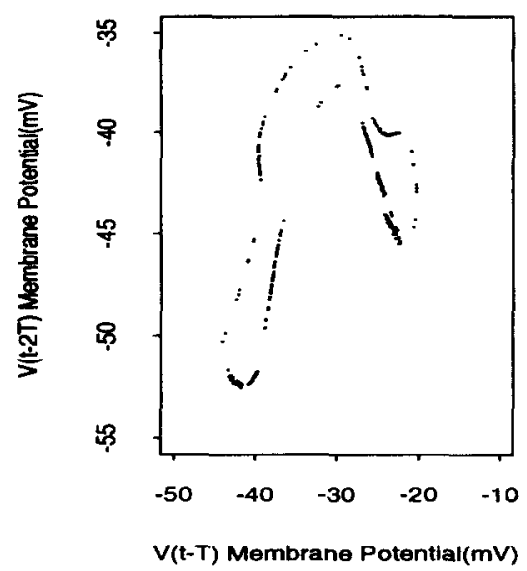

Fig. 5. Poincaré sections computed at coupling values of: (A) $146 \mathrm{pS}$ (period-4 oscillation); (B) 148 pS (period-8 oscillation; (C) $148.3 \mathrm{pS}$ (period-16 oscillation); and (D) $152 \mathrm{pS}$ (chaotic motion).

288.6 and $19.1 \mathrm{~ms}$, respectively. Figure 6(B) shows instantaneous period throughout the mesh at time $t=2.515 \mathrm{~s}$ when cell-to-cell coupling is set to $220 \mathrm{pS}$. The mean and standard deviation of these data are 282.3 and $2.7 \mathrm{~ms}$, respectively. Thus, a cell-to-cell coupling value of $220 \mathrm{pS}$ reduces standard deviation in oscillation period by a factor of seven. This trend continues as coupling is increased. In Fig. $6(\mathrm{C})-(\mathrm{D})$, periods are 280.3 and $278.5 \mathrm{~ms}$, and standard deviations are 0.6 and $0.2 \mathrm{~ms}$. Clearly, very little coupling between cells is required to achieve a remarkable degree of frequency entrainment, even in large networks.

Figure 7 shows results of a more realistic model of the sinus node in which oscillation properties of cells differ as a function of location within the node. The slowest oscillating cells are located in the center, and the fastest cells in the periphery of the mesh [1]. Instantaneous oscillation period is shown as a function of location in Fig. 7(A). These data were computed from a simulation in which $G_{\mathrm{c}}$ was set to zero throughout the mesh. Note that the range of oscillation periods is $320-380 \mathrm{~ms}$. Thus, oscillation period is slowed by about $30 \mathrm{~ms}$ relative to the data of Kodama and Boyett [1]. However, changes of 

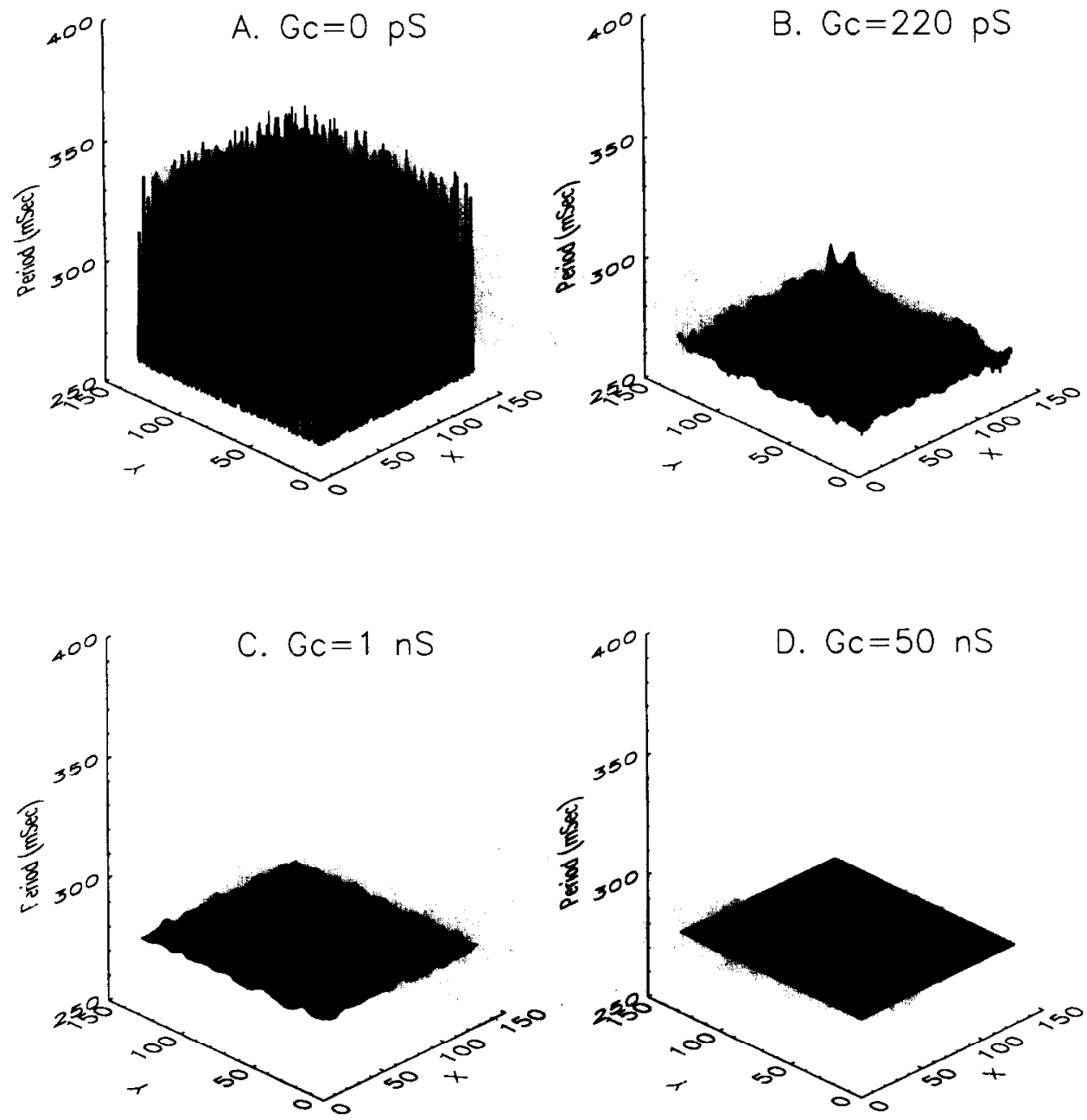

Fig. 6. Instantaneous period of oscillation ( $z$-axis) in a $128 \times 128$ mesh of SA node cells with randomly assigned oscillation period. Cell coupling is uniform throughout the mesh at values of (A) $0 \mathrm{pS},(\mathrm{B}) 220 \mathrm{pS}$, (C) $1 \mathrm{nS}$, and (D) $50 \mathrm{nS}$.

oscillation period and waveform as a function of location within the model node are similar to those shown by the Kodama-Boyett data.

Figure 7(B) shows instantaneous oscillation period computed $65 \mathrm{~s}$ after onset of the simulation, with $G_{\mathrm{c}}=250 \mathrm{pS}$. Period is virtually uniform throughout the mesh. It is interesting to note that approximate entrainment of oscillation frequency was achieved within a few hundred milliseconds for the data in Fig. 6, whereas entrainment required many tens of seconds in the model described for Fig. 7. Additionally, as we have noted previously $[11,12,41,42]$, models of the type described for Fig. 7 initiate the pacemaker potential in the peripheral regions of the node, with the wave of depolarization propagating inwards. This is precisely the behavior observed in the real SA node when it is isolated from the atrium [43]. Embedding the model SA node within a large mesh of model atrial cells shifts the site of pacemaker potential generation away from the periphery and towards the center of the node, and leads to outward propagation of the pacemaker potential and excitation of the atrium [11,12]. This makes clear the important role of atrial boundary conditions in constructing a physiologically realistic model of the SA node. 


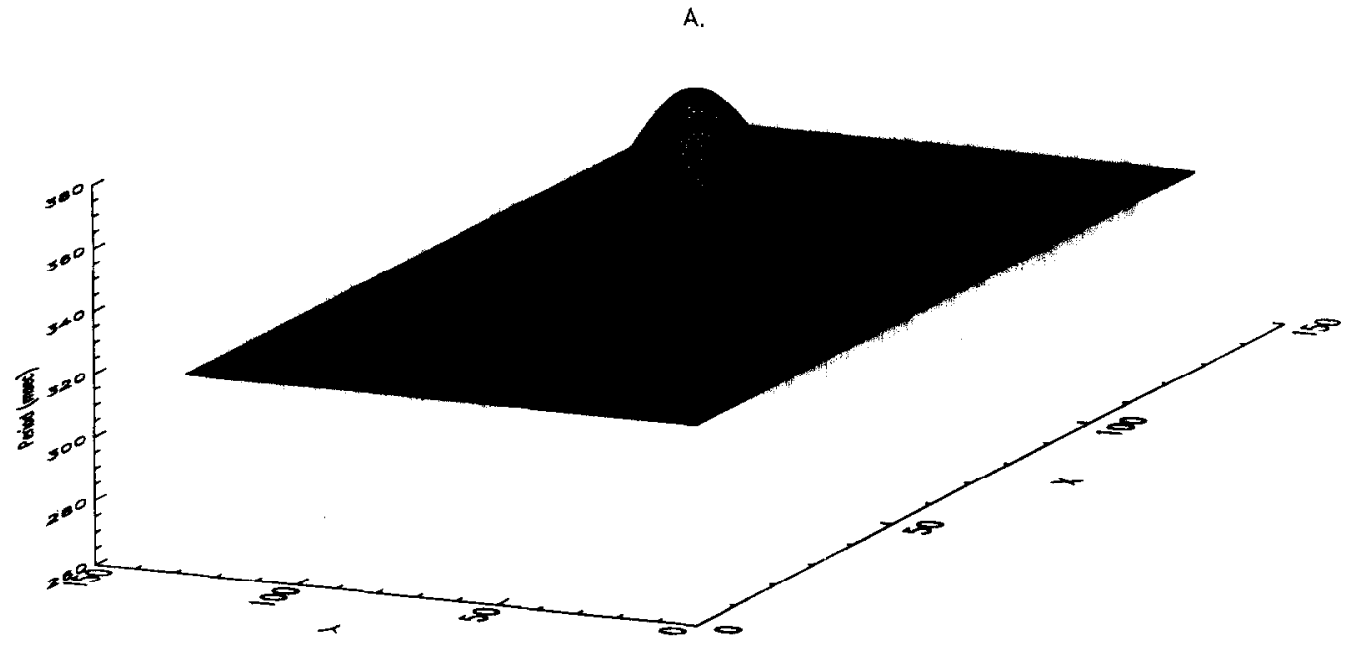

B.

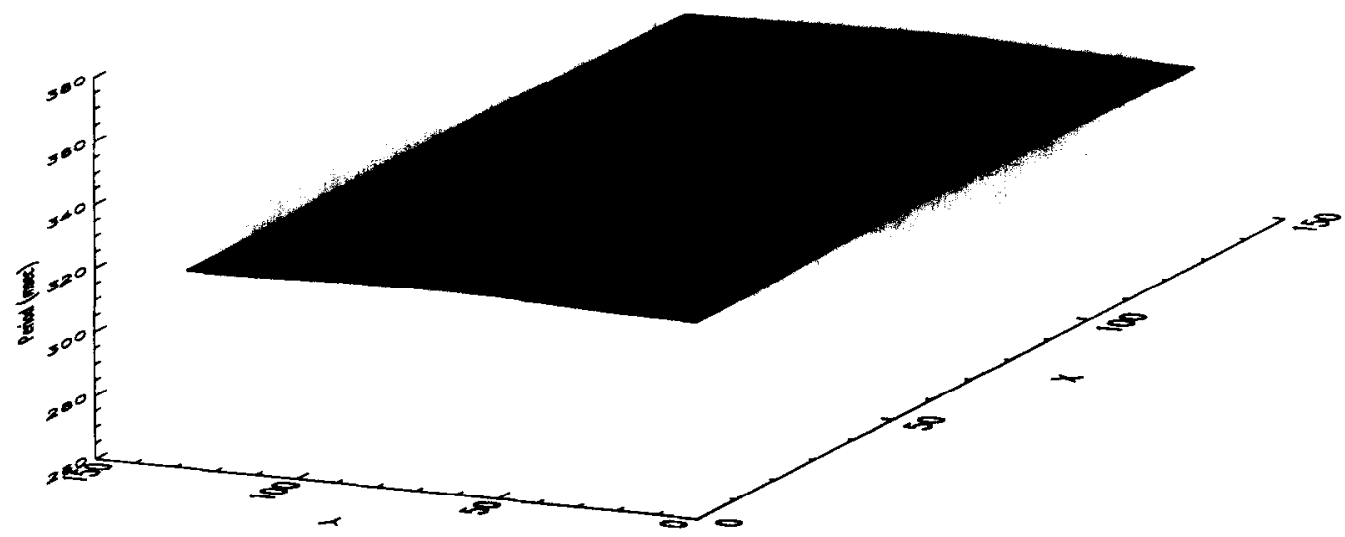

Fig. 7. (A) Distribution of oscillation period ( $z$-axis) in a $138 \times 128$ cell SA node model computed using $G_{\mathrm{c}}=0 \mathrm{pS}$. (B) Distribution of instantaneous period ( $z$-axis) in this same model at $t=65 \mathrm{~s}$ for $G_{\mathrm{c}}=250 \mathrm{pS}$.

\subsection{Abnormal pacemaking}

In this section we investigate properties of abnormal pacemakers induced by $\mathrm{Na}$ overloading subsequent to long-term $\mathrm{Na}-\mathrm{K}$ pump inhibition in single atrial cells and atrial networks, and the effect of gap junction conductance magnitude on the generation and propagation of this ectopic activity.

Figure $8(\mathrm{a})$ shows a train of spontaneous action potentials (solid line; left ordinate, $\mathrm{mV}$ ) and $\mathrm{Ca}$ transients (dashed line; right ordinate, $\mathrm{nM}$ ) computed using the single atrial cell model during conditions of $\mathrm{Na}$ overload. In order to induce $\mathrm{Na}$ overload, the peak $\mathrm{Na}-\mathrm{K}$ pump current was inhibited by $90 \%$ at time $t=0$. The cell was then stimulated with $1 \mathrm{nA}$, $2 \mathrm{~ms}$ duration inward current pulses at intervals of $2 \mathrm{~s}$ during the interval from $0-300 \mathrm{~s}$. At the end of this interval $[\mathrm{Na}]_{\mathrm{i}}$ had risen to $16.24 \mathrm{mM}$. Integration was continued for another 


\section{Single Atrial Cell}

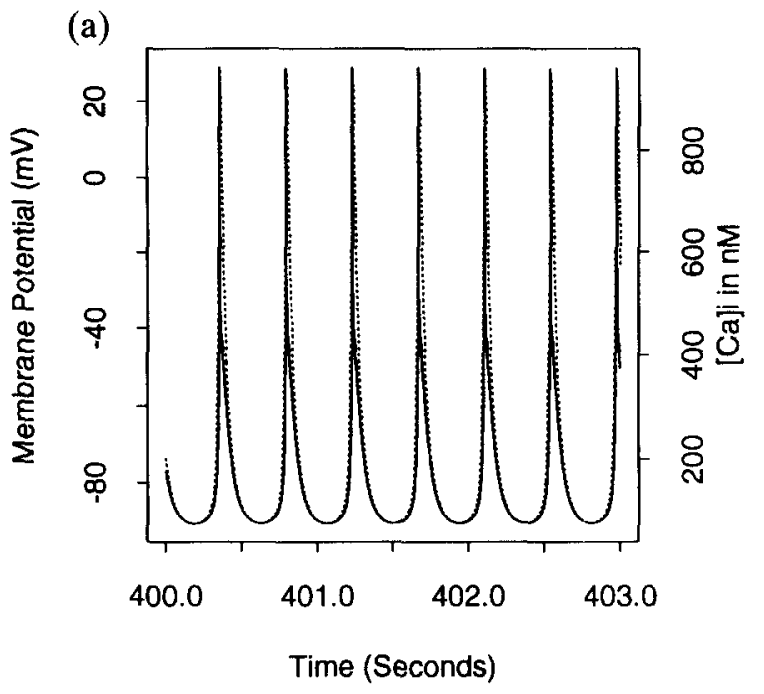

(b)

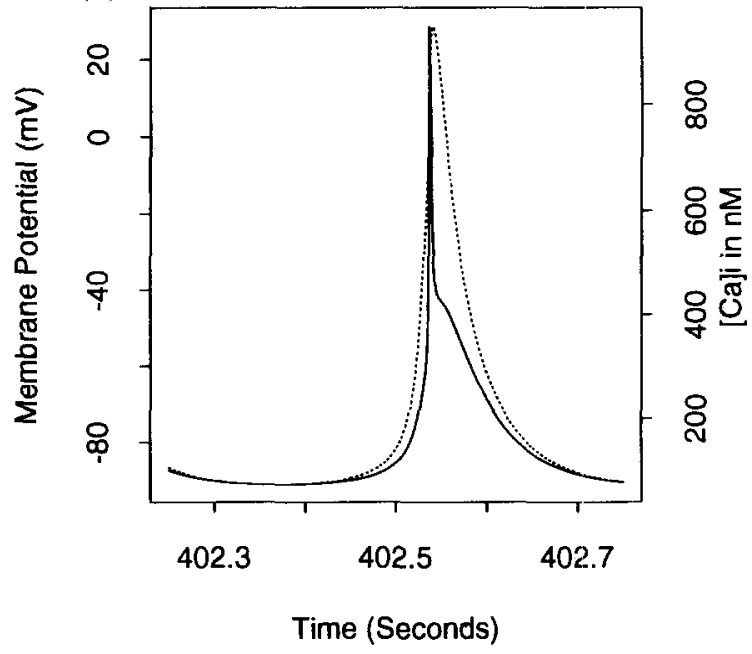

Fig. 8. (a) Spontaneous action potentials evoked by long term $\mathrm{Na}-\mathrm{K}$ pump inhibition ( $90 \%$ ) in a single atrial cell model (OXSOFT Heart V3.8). Membrane potential shown by the solid line (left ordinate, $\mathrm{mV}$ ) and [Ca] $]_{i}$ by the dashed line (right ordinate, nM). Time is in units of seconds (abscissa). (b) Spike 6 of Fig. 8(a) shown on an expanded timescale.

$100 \mathrm{~s}$ after cessation of the current pulse stimuli. The cell continued to oscillate during this interval. The data shown in Fig. 8 are the final $3 \mathrm{~s}$ of oscillation, from $t=400-403 \mathrm{~s}$. During this time, the Ca concentration oscillates between a minimum value of about $64 \mathrm{nM}$ to a peak value of $962 \mathrm{nM}$. The oscillation period is $438 \mathrm{~ms}$. Theoretical analyses of the DiFrancesco-Noble [24] Purkinje fiber equations have shown that at this level of internal $\mathrm{Na}$, the Ca subsystem undergoes a stable periodic oscillation. This theoretical result, in conjunction with the greater than $100 \mathrm{~s}$ numerically evaluted duration of oscillation suggests 
that the data of Fig. 8(a) correspond to a stable, sustained, periodic oscillation of $[\mathrm{Ca}]_{\mathrm{i}}$ in the single atrial cell model. Figure $8(\mathrm{~b})$ shows a higher resolution view of the 6th voltage and $\mathrm{Ca}$ spike shown in Fig. 8(a). Note that the $\mathrm{Ca}$ spike leads the voltage spike by about $15 \mathrm{~ms}$.

Figure 9(a) shows membrane potential (solid line; left ordinate, $\mathrm{mV}$ ) and internal $\mathrm{Ca}$

\section{Network Atrial Cell}
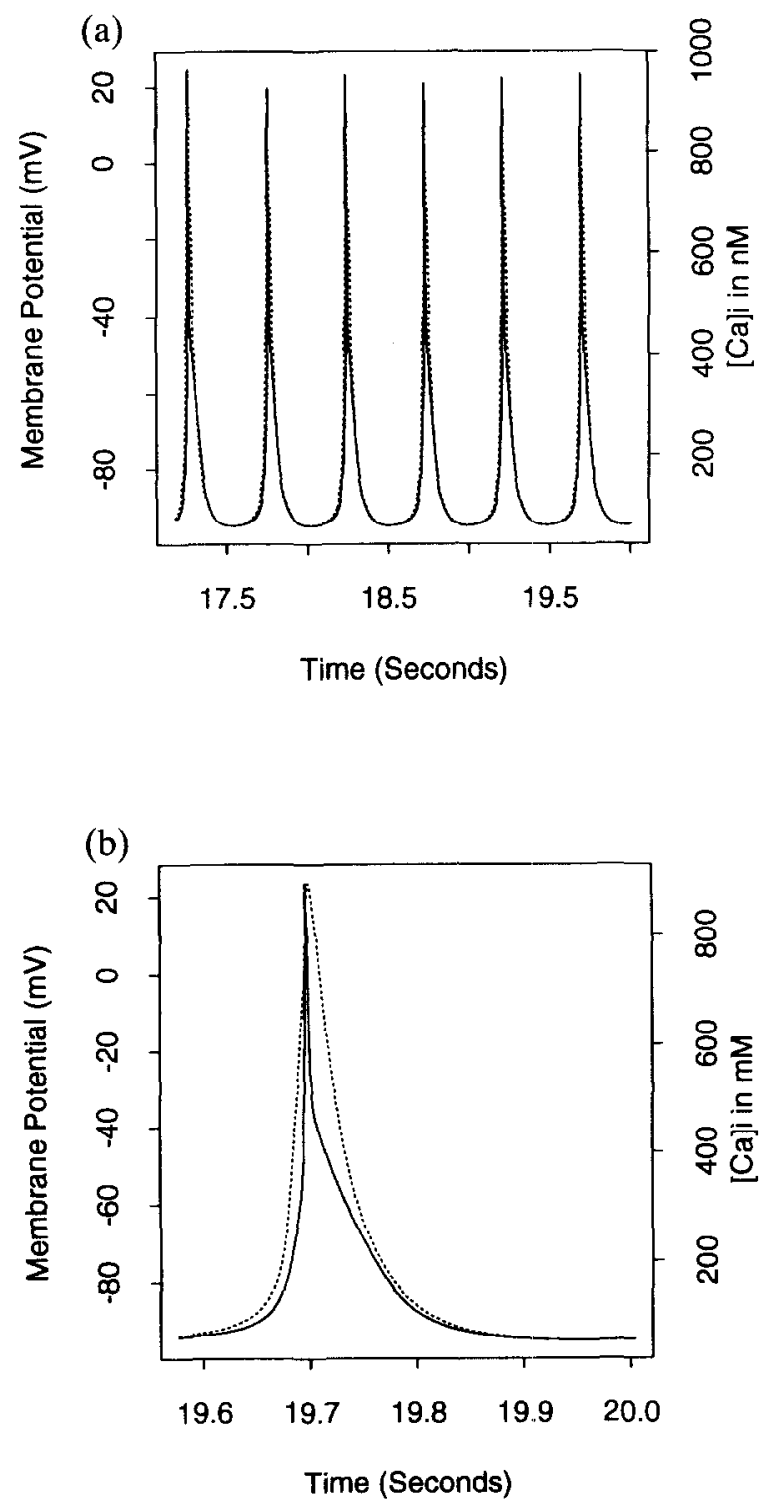

Fig. 9. (a) Spontaneous action potentials evoked by long term $\mathrm{Na}-\mathrm{K}$ pump inhibition (90\%) in a single cell positioned in the center of an atrial network simulated on the CM-5. Network size was $512 \times 512$ cells. Region of $\mathrm{Na}$ overload was a circular region of about 1000 cells positioned in the center of the array. The initial condition for perturbed network cells is the state of the single cell in Fig. 8 at $t=400 \mathrm{~s}$. Network cell-to-cell coupling was set to a uniform value of $1 \mu \mathrm{S}$, yielding a planar conduction velocity of $60 \mathrm{~cm} \mathrm{~s}^{-1}$. Membrane potential shown by the solid line (left ordinate, $\mathrm{mV}$ ) and $[\mathrm{Ca}]_{\mathrm{i}}$ by the dashed line (right ordinate, $\mathrm{nM}$ ). Time is in units of seconds (abscissa). (b) Spike 6 of Fig. 9(a) shown on an expanded timescale. 


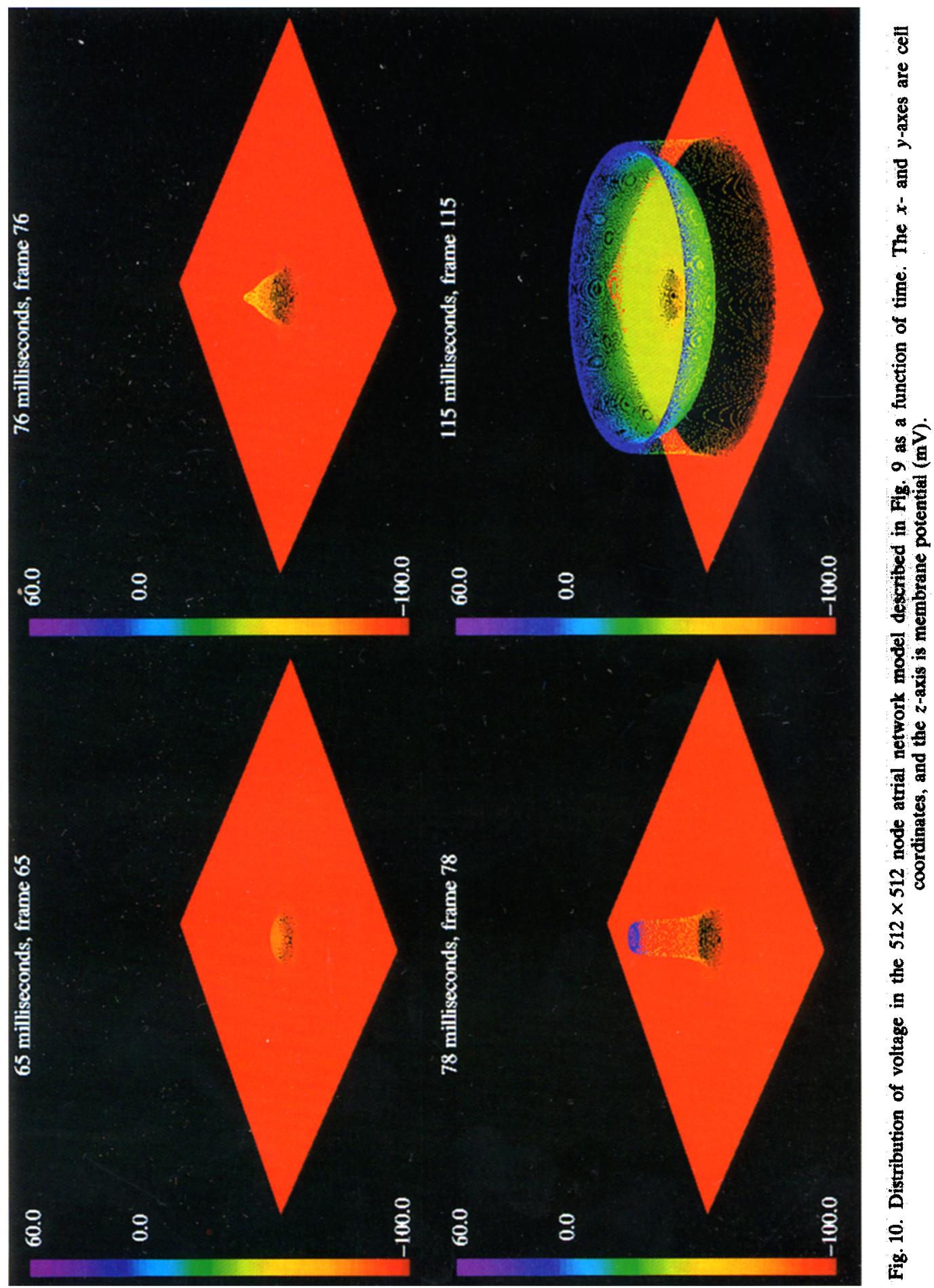




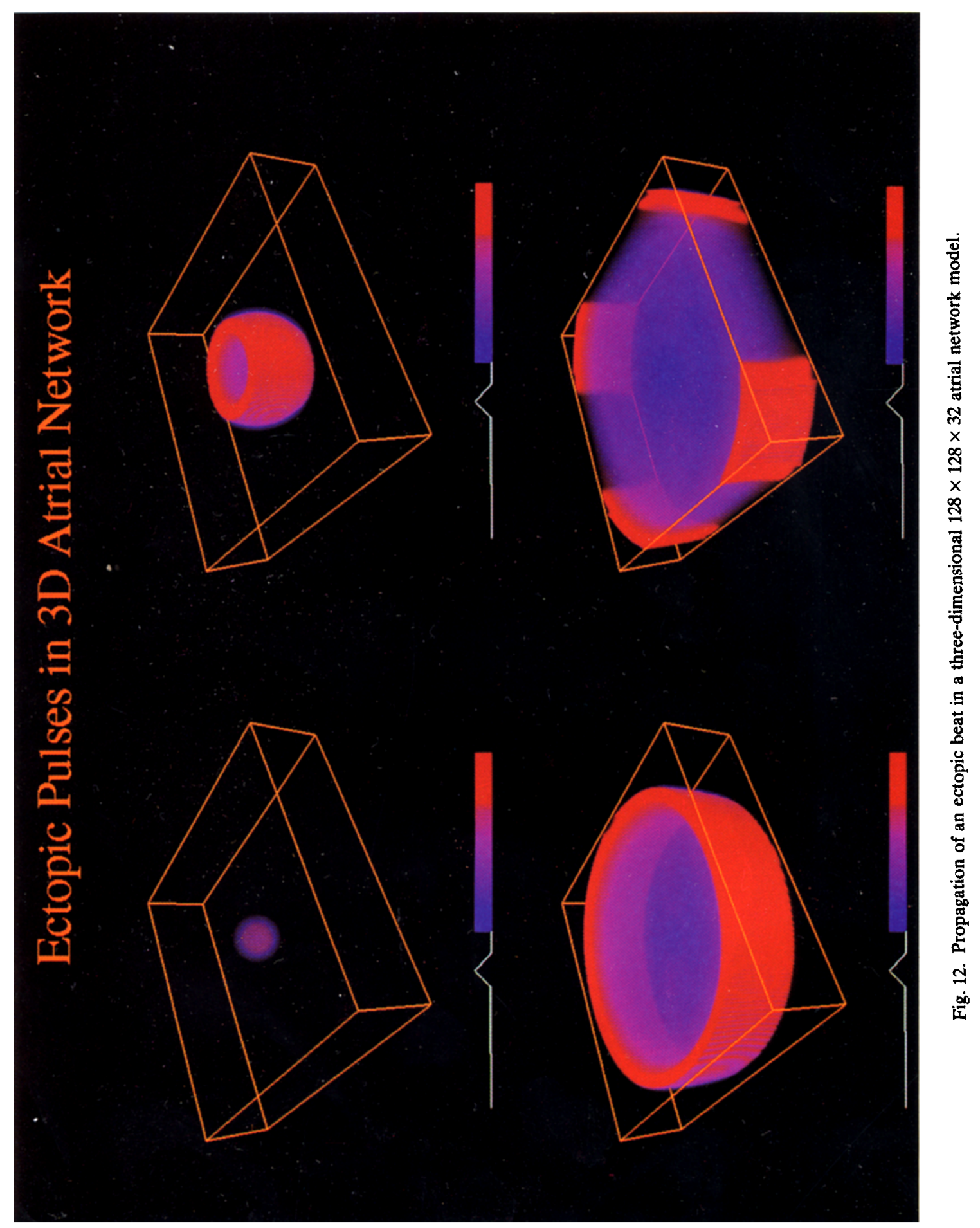


concentration (dashed line; right ordinate, $\mathrm{nM}$ ) as a function of time for a single cell within a $512 \times 512$ two-dimensional lattice of atrial cell simulated on the CM-5. This particular cell was positioned at the center of a circular region of cells (radius 26 cells) in which initial conditions corresponding to $\mathrm{Na}$ overload were imposed; the $\mathrm{Na}$-overloaded region was also positioned in the center of the $512 \times 512$ atrial mesh. The initial conditions were those obtained at $t=400 \mathrm{~s}$ for the single cell simulation described previously. Initial conditions for the remaining, quiescent network cells were set to their steady-state values. Cell-to-cell coupling was uniform at the value of $1 \mu \mathrm{S}$. This particular coupling conductance was selected because it yields an atrial plane wave conduction velocity of $60 \mathrm{~cm} \mathrm{~s}^{-1}$, a value similar to that observed within the atria [43]. Network activity was simulated for a total of $20 \mathrm{~s}$. Data from the final $3 \mathrm{~s}$ of the simulation are shown.

These data show a sustained oscillation of the central cell in the $\mathrm{Na}$-overloaded region. Here the term 'sustained' means that spontaneous oscillation continued for $20 \mathrm{~s}$ with no evidence of failure of conduction of the ectopic beats into the normal atrial cells or of systematic decrease in the overshoot potential of the cells. The slight variation in amplitude of the action potentials and the Ca-transients is an artifact resulting from storing data points at $1 \mathrm{~ms}$ intervals, and does not indicate any systematic decrease in overshoot amplitude that might indicate damping of the oscillation. Calcium concentration ranges from $62 \mathrm{nM}$ to $964 \mathrm{nM}$, and the period of oscillation is $485 \mathrm{~ms}$. Subtle differences in oscillation waveform, and the longer oscillation period relative to that in Fig. 8 are due to current shunting by the surrounding, more hyperpolarized normal atrial cells [12]. A higher resolution view of the 6th spike from the data of Fig. 9(a) is shown in Fig. 9(b). Note again that the Ca-spike leads the voltage spike.

The propagating nature of the abnormal pacemaker wave is shown in Fig. 10. Each panel shows the distribution of voltage for each cell in $512 \times 512$ atrial lattice at a particular instant of time. Figure 10(a)-(c) shows initiation of the first spike within the region of $\mathrm{Na}$ overload. Figure $10(\mathrm{~d})$ shows that the ectopic pacemaker wave has propagated beyond the region of $\mathrm{Na}$ overload to excite the surrounding normal atrial cells.

For a Na-overload region of size $N$, there will be a range of small cell-to-cell coupling values at which cells within the region of $\mathrm{Na}$ overload oscillate, but do not drive the surrounding normal atrial cells. At this same $N$, there may be values of $G_{\mathrm{c}}$ which are sufficiently large so that the shunting of current from the Na-overloaded region by the surrounding normal atrial cells prevents oscillation. As a consequence, for any $N$, there may be a range of intermediate coupling values that support the generation and propagation of ectopic beats. There may also be values of $N$ too small to support propagating ectopic beats at any value of $G_{c}$.

This relationship between $N$ and $G_{\text {c }}$ was explored numerically for two-dimensional lattice models in a series of simulations on the CM-5. The results are summarized in Fig. 11. The abscissa of this figure plots the number of cells within a circular region in the center of the atrial mesh in which Na-overloading was imposed. The ordinate plots the coupling conductance used in the simulations. For each $G_{\mathrm{c}}-N$ pair, network activity was simulated for $20 \mathrm{~s}$ in order to determine whether or not beating activity would be sustained. The filled triangles indicate $G_{c}-N$ pairs which did not support the generation and propagation of ectopic beats, indicated by the failure of the ectopic focus to excite the surrounding atrial cells or by a sudden or gradual decay of action potential overshoot potential during the course of the simulation. The open squares mark pairs which did support sustained generation and propagation of ectopic beats.

As predicted, at any $N$, except for values large relative to the total number of cells in the network, there is an upper and lower bound on values of $G_{c}$ that support propagating ectopic beats. Also note that cell-to-cell coupling values less than about $3 \mathrm{nS}$ do not 


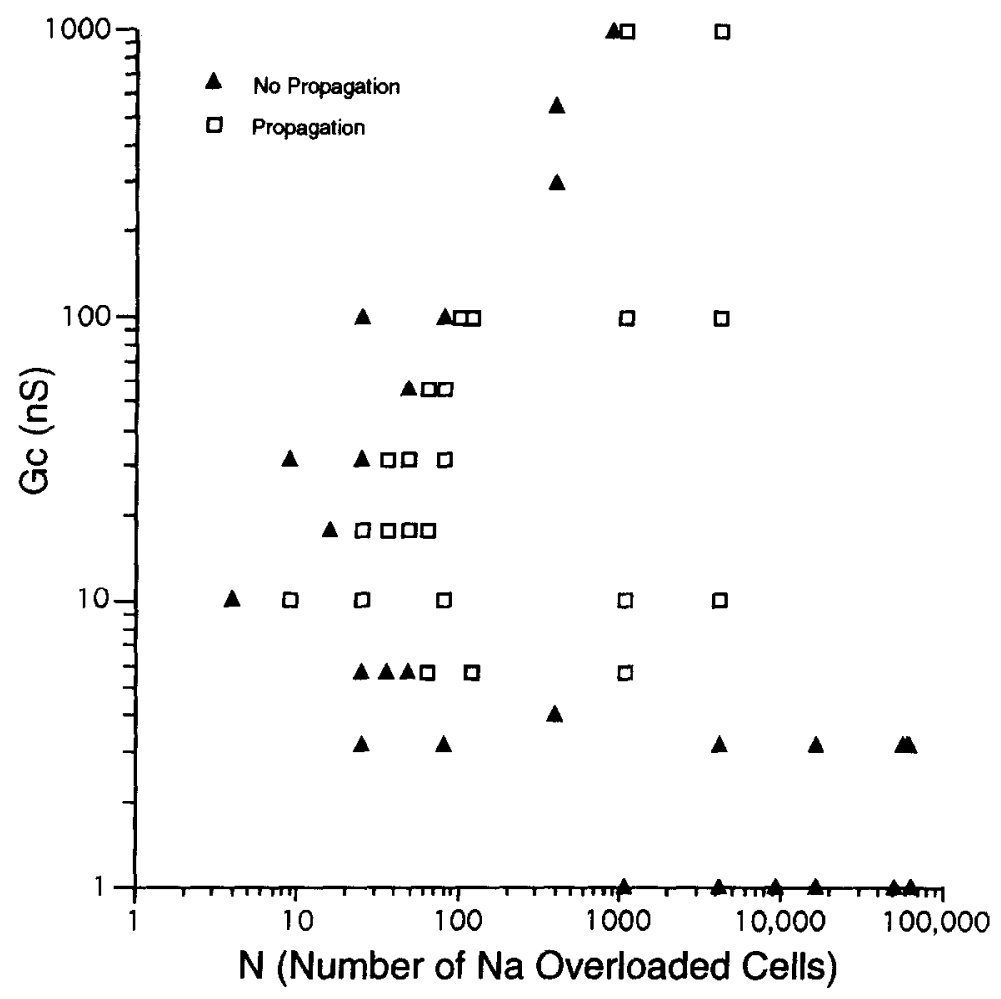

Fig. 11. Results of varying the number of centrally positioned Na-overloaded cells ( $N$, abscissa) and cell-to-cell coupling $\left(G_{\mathrm{c}}\right.$, ordinate, $\left.\mathrm{nS}\right)$ in a $512 \times 512$ atrial network model. Filled triangles mark $G_{\mathrm{c}}-N$ pairs for which spontaneous, propagating beats were not obtained. Open squares mark $G_{\mathrm{c}}-N$ pairs producing spontaneous propagating beats.

support the propagation of ectopic beats for any size region of $\mathrm{Na}$ overload (up to the maximum $N=64,516$ cells tested).

The data in Fig. 11 can be used to determine the minimum size region of Na overload that can support the generation and propagation of ectopic beats within two-dimensional atrial network models given an estimate of atrial cell-to-cell coupling. Conduction velocity in the atrium is known to be about $60 \mathrm{~cm} \mathrm{~s}^{-1}$ in the direction parallel to the long axis of each cell [43], and is dependent on the magnitude of cell-to-cell coupling. In our model, an atrial conduction velocity of $60 \mathrm{~cm} \mathrm{~s}^{-1}$ is achieved with a cell-to-cell coupling value of $1 \mu \mathrm{S}$. From Fig. 11, at this level of coupling, only about $1000 \mathrm{Na-overloaded} \mathrm{cells} \mathrm{are} \mathrm{required} \mathrm{to}$ sustain a propagating ectopic beat within a two-dimensional network.

Ectopic activity can also be generated and sustained as a result of Na-overloading in small groups of cells within three-dimensional networks. This is illustrated in Fig. 12, which shows data generated using a $128 \times 128 \times 32$ atrial network model with uniform coupling at $G_{\mathrm{c}}=1 \mu \mathrm{S}$ in which Na-overloading was induced in a central spherical volume of cells, 20 cells in diamcter. An alpha (white line) and color map arc shown below each panel, and indicate the degree of transparency and color-coding of the voltage scale for voltages ranging from the MDP to UV potentials. The sharp peak in the alpha-map indicates that transparency has been adjusted so that only a narrow range of depolarized voltages (coded by red) are highly visible. This makes it possible to visualize a 'shell' of depolarization, corresponding to the wavefront and waveback, propagating outward from the center of the ectopic focus. This activity was sustained for the time course of the simulation $(20 \mathrm{~s})$. 


\section{DISCUSSION}

The primary finding of this study is that coupling-conductance magnitude is a major determinant of the dynamics of coupled SA node cells. A total of five regimes of behavior can be defined on the basis of the two cell studies. Cells beat independently for $G_{\mathrm{c}}<1 \mathrm{pS}$. A regime of alternating quasiperiodic and periodic motions is found for $1 \leqslant G_{\mathrm{c}}<116 \mathrm{pS}$. Several bands of periodic orbits which undergo period-doubling bifurcation to chaos exist for $116 \leqslant G_{\mathrm{c}}<212 \mathrm{pS}$. Cells are frequency entrained for $G_{\mathrm{c}} \geqslant 212 \mathrm{pS}$, and waveform entrained for $G_{\mathrm{c}}<50 \mathrm{nS}$. Perhaps the most important finding is that all these dynamics, including frequency entrainment, occur at extremely low levels of coupling. Assuming a per channnel conductance of $50 \mathrm{pS}[40,44]$, all these regimes occur over a range corresponding to as few as one to five channels between each cell.

The finding that small coupling conductance can support frequency entrainment is consistent with recent work of Herve et al. [45] showing that frequency entrainment occurs in ventricular myocyte pairs at coupling conductances as low as $1 \mathrm{nS}$. Recent measurements of coupling conductance between pairs of SA node cells dissociated from the node indicate a coupling value of $2.5 \mathrm{nS}$, or about 50 channels [44]. Our results demonstrate that this level of coupling is about 10 times greater than that required for frequency entrainment. Thus, coupling in the normal SA node may be sufficiently large to assure an order of magnitude safety margin for frequency entrainment. However, gap-junction channel conductance is modulated by factors such as $\mathrm{pH}$ and internal calcium $(\mathrm{Ca})$ concentration $[46,47]$. In particular, Noma and Tsuboi [46] have demonstrated an order of magnitude reduction of gap-junction conductance between cardiac myocytes upon increasing cytosolic Ca-levels from about 40 to $400 \mathrm{nM}$. Therefore, factors which act to increase intracellular Ca-levels could produce reductions of gap-junction conductance. One consequence of ischemia in cardiac tissue is an increase in intracellular Ca-levels that occurs over a time course of tens of minutes $[48,49]$. If these increases in intracellular $\mathrm{Ca}$ are sufficiently high, then it is possible that coupling conductance could slowly drift through the region of complex dynamics we have explored.

We have demonstrated that Na-overloading in response to spatially localized $\mathrm{Na}-\mathrm{K}$ pump inhibition can induce propagating ectopic beats in two- and three-dimensional models of atrial networks. The size of the Na-overload region generating propagating ectopic beats depends on the cell-to-cell coupling magnitude. At a realistic coupling value yielding an atrial conduction velocity of $60 \mathrm{~cm} \mathrm{~s}^{-1}$, a surprisingly small perturbed region (about 1000 cells) can become an ectopic focus within a two-dimensional network.

There is substantial evidence indicating that $[\mathrm{Na}]_{\mathrm{i}}$ measured in whole heart increases over a time course of tens of minutes during experimentally induced global ischemia (perfused ferret heart, Pike et al. [50]; rat heart, Malloy et al. [51]; Tani and Neely [52]). Malloy et al. [51] measured control levels of $[\mathrm{Na}]_{1}$ using atomic spectroscopy $(6.2 \mathrm{mM})$. They then measured $[\mathrm{Na}]_{i}$ during global ischemia using $\mathrm{Na} \mathrm{NMR}$ spectroscopy, and saw a linear increase of $[\mathrm{Na}]_{i}$ at a rate of $0.43 \mathrm{mM} \mathrm{min}^{-1}$. Na levels reached $9.5 \mathrm{mM}$ after $10 \mathrm{~min}$. Pike et al. [50] also observed a linear increase in $[\mathrm{Na}]_{\mathrm{i}}$ during global ischemia, with $[\mathrm{Na}]_{\mathrm{i}}$ increasing by more than five-fold after $20 \mathrm{~min}$. Na levels may therefore reach the range of values required to evoke spontaneous activity of the type seen in this model after 15-20 min of global ischemia. Large increases in $[\mathrm{Ca}]_{i}$ are also observed during global ischemia (rat heart, Steenbergen et al. [48]). The increase in $[\mathrm{Ca}]_{i}$ is somewhat delayed with respect to those of $[\mathrm{Na}]_{\mathrm{i}}$ but still occurs over a similar time course of tens of minutes $[48,49]$.

The mechanisms giving rise to $\mathrm{Na}$ accumulation during global ischemia are not yet clear. Three can be identified: (a) $\mathrm{Na}$ entry through Na-permeable membrane channels; (b) $\mathrm{Na}$ 
entry via the $\mathrm{Na}-\mathrm{H}$ exchanger; and (c) reduction of Na-efflux through reductions in $\mathrm{Na}-\mathrm{K}$ pumping. Pike et al. [50] noted that mechanism (a) is unlikely since membrane depolarization occurs rapidly following onset of global ischemia. This would inactivate Na-channels, and reduce inward Na-flux. They have argued that mechanisms (b) and (c) are more likely. During global ischemia, increases in $[\mathrm{Na}]_{i}$ are accompanied by decreases in $\mathrm{pH}$, suggesting that the $\mathrm{Na}-\mathrm{H}$ exchanger is extruding $\mathrm{H}$-ion at the expense of increased intracellular Na-levels. Finally, Pike et al. [50] argued that, because of the small dissociation constant for the Na-K-ATPase, ATP depletion itself was unlikely to be the cause of pump inhibition. Rather, they argued that accumulation of inorganic phosphate during ischemia produces a decrease in the free energy of hydrolysis of ATP, thus inhibiting $\mathrm{Na}-\mathrm{K}$ pump function.

Our model predicts that Na-overloading in spatially localized regions of tissue can induce spontaneous ectopic beats. However, the model only captures properties of one possible mechanism of Na-overloading during ischemia. Modeling the detailed temporal evolution of cardiac cell dynamics during ischemia will undoubtedly require incorporation of the $\mathrm{Na}-\mathrm{H}$ exchanger into the single cell and network models. Finally, one critical aspect of modeling the development of $\mathrm{Na}$ overload within networks has been omitted from our model. That aspect is flux of ions through gap junction channels. Na accumulation within network cells will reflect a competition between two competing processes: (a) accumulation of $\mathrm{Na}$ due to inhibition of $\mathrm{Na}-\mathrm{K}$ pumping or by alterations of $\mathrm{Na}-\mathrm{H}$ exchanger function; and (b) efflux of $\mathrm{Na}$ through gap-junction channels to neighboring cells. The time course of $\mathrm{Na}$ accumulation within cell networks will be highly dependent on the balance achieved between these two processes.

Acknowledgements-Supported by NSF grants DIR 91-17874, The Whitaker Foundation, NSF Research Associateship ASC-9211131, and U.S. Army Research Office contract DAAL03-89-C-0038.

\section{REFERENCES}

1. I. Kodama and M. Boyett, Regional differences in the electrical activity of the rabbit sinus node, Pflugers Arch. 404, 214-226 (1985).

2. H. Honjo and M. R. Boyett, Correlation between action potential parameters and the size of single sino-atrial node cells isolated from the rabbit heart, $J$. Physiol. 452, 128P (1992).

3. D. L. Ypey, D. E. Clapham and R. L. DeHaan, Development of electrical coupling and action potential synchrony between paired aggregates of embryonic heart cells, Membrane Biol. 51, 75-96 (1979).

4. R. L. DeHaan, D. L. Y. E. H. Williams and D. E. Clapham, Intercellular coupling of embryonic heart cells, in Mechanisms of Cardiac Morphogenesis and Teratogenesis, edited by T. Pexieder, pp. 299-316. Raven, New York (1981).

5. R. L. DeHaan, In vitro models of entrainment of cardiac cells, in Cardiac Rate and Rhythm, edited by L. N. Bouman and H. J. Jongsma, pp. 323-361. Nijhoff, Amsterdam (1982).

6. J. Jalife, Mutual entrainment and electrical coupling as mechanisms for synchronous firing of rabbit sino-atrial pace-maker cells, J. Physiol. 356, 221-243 (1984).

7. M. Delmar, J. Jalife and D. C. Michaels, Effects of changes in excitability and intercellular coupling on synchronization in the rabbit sino-atrial node, J. Physiol. 370, 127-150 (1986).

8. V. Torre, A theory of synchronization of heart pace-maker cells, J. Theor. Biol. 61, 55-71 (1976).

9. K. Yanagihara, A. Noma and H. Irisawa, Reconstruction of sinoatrial node pacemaker potential based on the voltage-clamp experiments, Jap. J. Physiol. 30, 841-857 (.980).

10. D. C. Michaels, E. P. Matyas and J. Jalife, Dynamic interactions and mutual synchronization of sinoatrial node pacemaker cells, Circ. Res. 58, 706-720 (1986).

11. R. L. Winslow, A. Varghese, D. Noble, J. C. Denyer and A. Kimball, Modelling large SA node-atrial cell networks on a massively parallel processor, J. Physiol. 446, 242P (1992).

12. R. L. Winslow, A. Kimball, A. Varghese and D. Noble, Simulating cardiac sinus and atrial network dynamics on the connection machine, Physica D64(3), 281-298 (1993).

13. D. Cai, R. L. Winslow and D. Noble, Effects of gap junction conductance on oscillation properties of coupled sinoatrial node cells, Computer in Cardiology Proc. Durham, North Carolina, Oct 1992, IEEE Computer Society Press (1995) in press.

14. D. Noble, D. DiFrancesco and J. Denyer, Ionic mechanisms in normal and abnormal cardiac pacemaker activity, in Neuronal and Cellular Oscillators, edited by J. W. Jacklet. Marcel Dekker, New York (1989). 
15. D. Noble, D. Difrancesco and J. Denyer, Ionic mechanisms in normal and abnormal cardiac pacemaker activity, in Neuronal and Cellular Oscillators edited by J. W. Jacklet. Marcel Dekker, New York (1989).

16. D. Noble, OXSOFT Heart Program Manual. OXSOFT Ltd., Oxford (1990).

17. C. S. Peskin, Mathematical Aspects of Heart Physiology. Courant Institute of Mathematical Sciences, New York (1975).

18. R. E. Mirollo and S. H. Strogatz, Synchronization of pulse-coupled biological oscillators, SIAM J.Appl. Math. 50, 1645-1662 (1990).

19. A. A. Brailove, The dynamics of two pulse-coupled relaxation oscillators, Int. J. Bifurcation Chaos 2 , 341-352 (1992).

20. G. R. Ferrier and G. I. Moe, Effects of calcium on acetylstrophanthidin induced transient depolarizations in canine Purkinje tissue, Circ. Res. 33, 508-515 (1973).

21. W. J. Lederer and R. W. Tsien, Transient inward current underlying arrhythmogenic effects of cardiotonic steroids in Purkinje fibres, J. Physiol. 263, 73-100 (1976).

22. R. S. Kass, R. W. Tsien and R. Weingart, Ionic basis of transient inward current induced by strophanthidin in cardiac Purkinje fibres, J. Physiol. 281, 209-226 (1978).

23. D. G. Allen, D. A. Eisner and C. A. Orchard, Characteristics of oscillations of intracellular calcium concentration in ferret ventricular muscle, $J$. Physiol. 352, 113-128 (1984).

24. D. DiFrancesco and D. Noble, A model of cardiac electrical activity incorporating ionic pumps and concentration changes, Phil. Trans. R. Soc. Lond. B307, 353-398 (1985).

25. D. W. Hilgemann and D. Noble, Excitation-contraction coupling and extracellular calcium transients in rabbit atrium: reconstruction of basic cellular mechanisms, Proc. R. Soc. Lond. B230, 163-205 (1987).

26. Y. E. Earm and D. Noble, A model of the single atrial cell: relation between calcium current and calcium release, Proc. $R$. Soc. Lond. B240, 83-96 (1990).

27. D. Noble, Ionic mechanisms determining the timing of ventricular repolarization: significance for cardiac arrhythmias, Ann. N.Y. Acad. Sci. 644, 1-22 (1992).

28. C.-H. Luo, A dynamic model of the mammalian ventricular action potential: formulation and physiological simulations, PhD thesis, Case Western Reserve University (1991).

29. C.-H. Luo and Y. Rudy, A model of the ventricular action potential: depolarization, repolarization, and their interaction, Circ. Res. 68, 1501-1526 (1991).

30. A. Varghese and R. L. Winslow, Dynamics of the ca subsystem in cardiac Purkinje fibers, Physica D (1993).

31. N. H. Packard, J. D. F. J. P. Crutchfield and R. S. Shaw, Geometry from a time series, Phys. Rev. Lett. 45, $712-716(1980)$.

32. F. Takens, Detecting strange attractors in turbulence, in Lecture Notes in Mathematics, Vol. 898, edited by D. A. Rand and L. S. Young. Springer, Berlin (1980).

33. P. Grassberger and I. Procaccia, Measuring the strangeness of strange attractors, Physica D9, 189-208 (1983).

34. H. L. Swinney, Observations of order and chaos in nonlinear systems, Physica D7, 3-15 (1983).

35. J. P. Eckmann and D. Ruelle, Ergodic theory of chaos and strange attractors, Rev. Mod. Phys. 57, 617-656 (1985).

36. T. S. Parker and L. O. Chua, Practical Numerical Algorithms for Chaotic Systems. Springer, New York (1989).

37. M. Kubicek and M. Marek, Computational Methods in Bifurcation and Dissipative Structures. Springer, New York (1983).

38. V. I. Arnold, Geometrical Methods in the Theory of Ordinary Differential Equations, Springer, New York (1983).

39. E. Ott, Chaos in Dynamical Systems. Oxford University Press, Oxford (1992).

40. M. B. Rook, H. J. Jongsma and A. C. G. van Ginnekan, Properties of single gap junctional channels between isolated neonatal rat heart cells, Am. J. Physiol. 255, H770-782 (1988).

41. R. L. Winslow, A. L. Kimball, D. Noble and J. C. Denyer, Computational model of the mammalian cardiac sinus node implemented on a connection machine cm-2, Med. Biol. Eng. Comp. 29(suppl 2), 832 (1991).

42. R. L. Winslow, A. Kimball, D. Noble and J. Denyer, Simulation of large-scale sinus node and atrial cell network models on the connection machine cm-2, J. Physiol. 438, 180P (1991).

43. C. J. H. J. Kirchof, The sinus node and atrial fibrillation, PhD thesis, University of Maastricht (1990).

44. J. Anumonwo, H. Z. Wang, E. Tranka-Janik, R. Dunham, M. Delmar and J. Jalife, Gap junctional channels in adult mammalian sinus nodal cells, Circ. Res. 71, 229-239 (1992).

45. J. C. Herve, D. Noble, B. Bastide, L. Cronier and J. Deleze, Nexus channel requirements for action potential entrainment in ventricular cardiac cells: simulation and experimental approaches, $J$. Physiol. 446, 343P (1992).

46. A. Noma and $\mathrm{N}$. Tsuboi, Dependence of junctional conductance on proton, calcium, and magnesium ions in cardiac paired cells of guinea-pig, $J$. Physiol. 382, 193-211, (1987).

47. D. C. Spray and J. M. Burt, Structure-activity relations of the cardiac gap junction channel, Am. J. Physiol. 258, C195-C205 (1990).

48. C. Steenbergen, E. Murphy, L. Levy and R. E. London, Elevation in cytosolic free calcium concentration in myocardial ischemia in perfused rat heart, Circ. Res. 60, 700-707 (1990).

49. E. Marban, M. Kitakaze, H. Kusuoka, J. K. Porterfield, D. T. Yue and V. P. Chacko, Intracellular free calcium concentration measured with ${ }^{19}$ F NMR spectroscopy in intact ferret hearts, Proc. Nat. Acad. Sci. 84, $6005-6009$ (1987).

50. M. M. Pike, M. Kitakaze and E. Marban, Na-NMR measurements of intracellular sodium in intact perfused ferret hearts during ischemia and reperfusion, Am. J. Physiol. 259, H1767-H1773 (1990). 
51. C. Malloy, D. Buster, M. Margarida, C. Castro, G. Geraldes, M. Jeffrey and A. Sherry, Influence of global ischemia on intracellular sodium in the perfused rat heart, Mag. Res. Med. 15, 33-44 (1990).

52. M. Tani and J. R. Neely, $\mathrm{Na}^{+}$accumulation increases $\mathrm{Ca}^{2+}$ overload and impairs function in anoxic rat heart, J. Mol. Cell Cardiol. 22, 57-72 (1990). 